\title{
Mössbauer rotor experiment as new proof of general relativity: Rigorous computation of the additional effect of clock synchronization
}

\author{
Christian Corda
}

June 20, 2019

International Institute for Applicable Mathematics and Information Sciences, B. M. Birla Science Centre, Adarshnagar, Hyderabad 500063 (India); e-mail address: cordac.galilei@gmail.com

\begin{abstract}
We received an Honorable Mention at the Gravity Research Foundation 2018 Awards for Essays on Gravitation by showing that a correct general relativistic interpretation of the Mössbauer rotor experiment represents a new, strong and independent, proof of Einstein's general theory relativity (GTR). Here we correct a mistake which was present in our previous computations on this important issue by deriving a rigorous computation of the additional effect of clock synchronization. Finally, we show that some recent criticisms on our general relativistic approach to the Mössbauer rotor experiment are incorrect, by ultimately confirming our important result.
\end{abstract}

\section{Introduction}

The Mössbauer effect was discovered by the German physicist R. Mössbauer in 1958 [1]. For this effect, Mössbauer was awarded the 1961 Nobel Prize in Physics. The effect consists in resonant and recoil-free emission and absorption of gamma rays, without loss of energy, by atomic nuclei bound in a solid. It is very important for various research fields in physics and chemistry. In this paper, the so called Mössbauer rotor experiment will be discussed, see Figure 1. In that case, the Mössbauer effect works by using an absorber orbited around a source of resonant radiation (or vice versa). The aim of the experiment is to verify the relativistic time dilation for a moving resonant absorber which generates a relative energy shift between emission and absorption lines. 


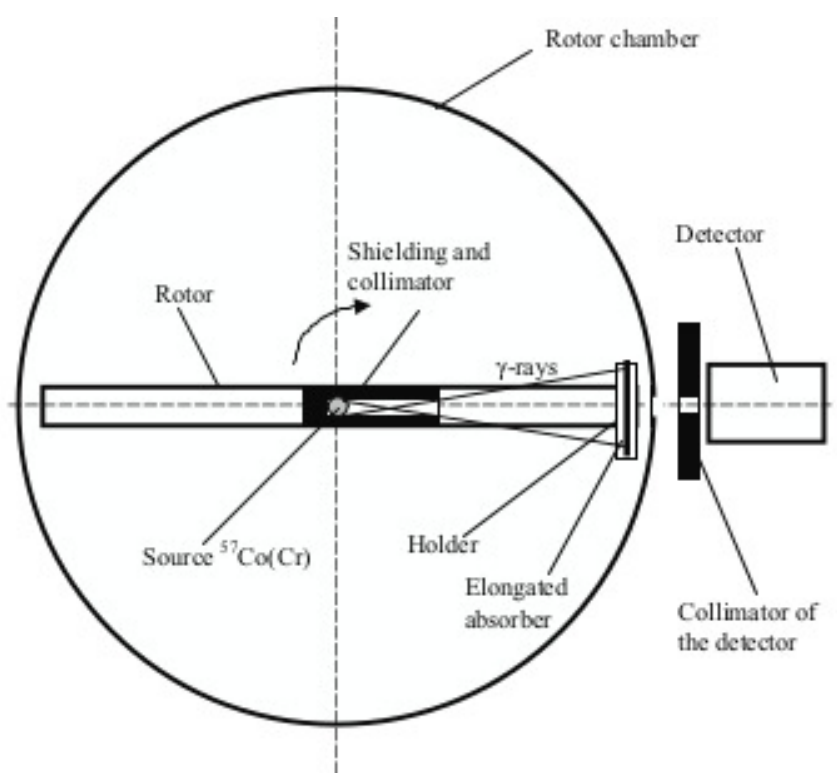

Figure 1: Scheme of the new Mössbauer rotor experiment, adapted from ref. 2]

The idea to use a general relativistic framework in order to explain the Mössbauer rotor experiment has a long history, which started from the famous book of Pauli [3]. The key point is Einstein's equivalence principle, (EEP) which states the equivalence between the gravitational "force" and the pseudo-force experienced by an observer in a non-inertial frame of reference [4-6]. The case of the rotating frame of reference of the Mössbauer rotor experiment is a particular case of the EEP [4 - 6]. This permits one to reanalyse the theoretical framework of the Mössbauer rotor experiment directly in the rotating frame of reference through a full general relativistic treatment [4 - 6].

A historical experiment on the Mössbauer rotor effect was due to Kündig [7. Kündig's work was recently reanalysed by a team of researchers in [2, 8]. Such a team, first reanalysed in 8 , the data of Kündig's experiment. Then, the team's researchers realized their own experiment on the time dilation effect in a rotating system [2]. In [8], the authors found that in the original experiment of Kündig [7] errors were present in the data processing. After the correction of the errors of Kündig, the experimental data gave the value [8]

$$
\frac{\nabla E}{E} \simeq-k \frac{v^{2}}{c^{2}},
$$

where $k=0.596 \pm 0.006$, instead of the standard general relativistic prediction $k=0.5$ due to time dilation [3]. This was a puzzling issue. In [8], the authors emphasized that, on one hand, the deviation of the coefficient $k$ in equation (1) from 0.5 exceeds by almost 20 times the measuring error. On the other hand, the revealed deviation cannot be attributed to the influence of rotor vibrations 
and/or other kinds of disturbing factors. The potential disturbing factors was indeed excluded by a very good methodology of Kündig [7]. Such a methodology is given by a first-order Doppler modulation of the energy of $\gamma$-quanta on a rotor at each fixed rotation frequency [7. Hence, the experiment of Kündig is today considered as being the most precise among other similar experiments [9 - 13]. The experimenters [9 - 13] indeed measured only the count rate of detected $\gamma$-quanta as a function of rotation frequency. In 8 it has also been shown that the experiment in [13] confirms the supposition $k>0.5$. Remarkably, the experiment in [13] contains much more data than the ones in [9-12]. In order to better investigating the results in 8 , the authors realized their own experiment [2. In [2], neither the scheme of the Kündig experiment [7, nor the schemes of other known experiments on the subject previously mentioned above [9 - 13] have been repeated. This permitted to obtain a completely independent information on the value of $k$ in Eq. (1). The authors refrained indeed from the first-order Doppler modulation of the energy of $\gamma$-quanta [2. This permitted to exclude the uncertainties in the realization of this method [2]. The standard scheme in [9 - 13] has been followed also in [2]. It means that the count rate of detected $\gamma$-quanta $N$ as a function of the rotation frequency $\nu$ has been measured. But, differently from the experiments [9 - 13], in [2] the influence of chaotic vibrations on the measured value of $k$ has been evaluated. A method involving a joint processing of the data collected for two selected resonant absorbers with the specified difference of resonant line positions in the Mössbauer spectra has been developed [2]. The final result was the value $k=0.68 \pm 0.03$ [2]. This confirms that the coefficient $k$ in Eq. (1) substantially exceeds 0.5 . The reader can see the scheme of the new Mössbauer rotor experiment in Figure 1. Further technical details on this scheme can be found in [2].

In [4 - 6] we reanalysed the theoretical framework of the Mössbauer rotor experiment directly in the rotating frame of reference through a full general relativistic treatment. We have shown that, in previous analyses in the literature, an important effect of clock synchronization has been missed. Thus, the correct general relativistic prevision gives $k \simeq \frac{2}{3}[4-6]$, which is in perfect agreement with the new experimental results in [2]. In other words, the general relativistic interpretation [4 - 6] shows that the new experimental results of the Mössbauer rotor experiment in [2] are a new, strong and independent, proof of the GTR. Remarkably, our results on the Mössbauer rotor experiment received an Honorable Mention at the Gravity Research Foundation 2018 Awards for Essays on Gravitation [4].

We must also stress that various papers in the literature (included ref. 9 published in Phys. Rev. Lett.) missed the additional effect of clock synchronization [2, 7 - 16]. This generated some claim of invalidity of relativity theory and/or some attempts to explain the experimental results through nonconventional or "exotic" effects [2, 8, 14, 15, 16]. One of these "exotic" effects will be partially discussed in Section 3 of this paper.

Here, an important mistake which was present in our previous computations on this important issue in [4 - 6] will be solved, by deriving a rigorous computation of the additional effect of clock synchronization. Finally, it will be 
shown that some recent criticisms [17, 18] on our general relativistic approach to the Mössbauer rotor experiment are incorrect, by ultimately confirming our important result.

\section{General relativistic interpretation of the Möss- bauer rotor experiment}

\subsection{The "gravitational blueshift"}

Following [4 - 6], one uses a transformation from an inertial frame, in which the space-time is Minkowskian, to a rotating frame of reference in cylindrical coordinates. In the starting inertial frame, one has the line-element [4 - 6]

$$
d s^{2}=c^{2} d t^{2}-d r^{2}-r^{2} d \phi^{2}-d z^{2}
$$

One considers the following transformation to a frame of reference $\left\{t^{\prime}, r^{\prime}, \phi^{\prime} z^{\prime}\right\}$ which rotates with an uniform angular rate $\omega$ with respect to the starting inertial frame $[4-6]$

$$
t=t^{\prime} \quad r=r^{\prime} \quad \phi=\phi^{\prime}+\omega t^{\prime} \quad z=z^{\prime} .
$$

Eq. (2) becomes the famous Langevin metric in the rotating frame [4-6]

$$
d s^{2}=\left(1-\frac{r^{\prime 2} \omega^{2}}{c^{2}}\right) c^{2} d t^{\prime 2}-2 \omega r^{\prime 2} d \phi^{\prime} d t^{\prime}-d r^{\prime 2}-r^{\prime 2} d \phi^{\prime 2}-d z^{\prime 2}
$$

Despite it is simple to grasp, the transformation (3) is highly illustrative of the GTR general covariance. It indeed shows that one can start to work in a "simpler" frame and then transforming to a more "complex" one [4 - 6]. Through the EEP, the line element (4) is interpreted in terms of a curved space-time in presence of a static gravitational field [4 - 6]. This gives a pure general relativistic interpretation of the pseudo-force that an observer in a rotating, non-inertial frame of reference experiences $[4-6]$. One sets the origin of the reference frames (both of the rotating and resting ones) in the source of the emitting radiation [4 6]. Thus, one gets a first contribution arising from the "gravitational blueshift" [4 - 6]. One easily computes this contribution by using Eq. (25.26) in [19], which, in the twentieth printing 1997 of [19], reads

$$
z \equiv \frac{\Delta \lambda}{\lambda}=\frac{\lambda_{\text {received }}-\lambda_{\text {emitted }}}{\lambda_{\text {emitted }}}=\left|g_{00}\left(r_{1}^{\prime}\right)\right|^{-\frac{1}{2}}-1 .
$$

This equation gives the redshift of a photon emitted by an atom at rest in a gravitational field and received by an observer at rest at infinity. Here, a slightly different equation with respect to Eq. (25.26) in [19] will be used. In fact, here one considers a gravitational field which increases with increasing radial coordinate $r^{\prime}$. Instead, Eq. (25.26) in [19] concerns a gravitational field which decreases with increasing radial coordinate $[4-6]$. The result will be a 
blueshift (a negative shift) rather than a redshift. In addition, we set the zero potential in $r^{\prime}=0$ rather than at infinity, and we use the proper time $\tau$ rather than the wavelength $\lambda[4-6]$. Therefore, from Eq. (4), one gets [4-6]

$$
\begin{aligned}
z_{1} \equiv \frac{\Delta \tau_{10}-\Delta \tau_{11}}{\tau_{1}} & =1-\left|g_{00}\left(r_{1}^{\prime}\right)\right|^{-\frac{1}{2}}=1-\frac{1}{\sqrt{1-\frac{\left(r_{1}^{\prime}\right)^{2} \omega^{2}}{c^{2}}}} \\
& =1-\frac{1}{\sqrt{1-\frac{v^{2}}{c^{2}}}} \simeq-\frac{1}{2} \frac{v^{2}}{c^{2}} .
\end{aligned}
$$

Here, $\Delta \tau_{10}$ is the delay of the emitted radiation, $\Delta \tau_{11}$ is the delay of the received radiation, $r_{1}^{\prime} \simeq c \tau_{1}$ is the radial coordinate of the detector and $v=r_{1}^{\prime} \omega$ is the tangential velocity of the detector $[4-6]$. For the sake of completeness, we clarify the meaning of "delay of the emitted and of the received radiation". We are referring to the issue that the proper time interval between events of emission of two photons as measured by the standard clock at the point of emission is different from the proper time interval between events of absorption of those photons as measured by identical standard clock at the point of absorption. This was the initial reasoning of Einstein in [28] and was elegantly adopted by Weyl in 29].

Then one obtains the first contribution, say $k_{1}=\frac{1}{2}$, to $k[4-6]$. Let us again emphasize that it is the power of the EEP which enabled us to use a pure general relativistic treatment in previous analysis $[4-6]$.

\subsection{Additional effect of clock synchronization: correcting an important mistake}

In order to understand the necessity of an additional effect, one stresses that the variations of proper time $\Delta \tau_{10}$ and $\Delta \tau_{11}$ have been calculated in the origin of the rotating frame which is located in the source of the radiation [4 - 6]. Then, the key point is that the detector moves with respect to the origin in the rotating frame $[4-6]$. This means that the clock in the detector, which is located in the laboratory frame at rest, has to be synchronized with the rotating clock in the origin. This generates a second, additional, contribution to time dilation [4-6], which was missed in previous analyses [2, 3, $7-18]$. In order to compute this second contribution, in $[4-6]$ we claimed to use Eq. (10) of 20, by stressing that it represents the proper time increment $d \tau$ on the moving clock having radial coordinate $r^{\prime}$ for values $v \ll c$. Actually, in our works [46] an important mistake was present. In fact, Eq. (10) of [20] is given by

$$
d \tau=d t^{\prime}-\frac{\omega r^{\prime 2} d \phi^{\prime}}{c^{2}}
$$

while in $[4-6]$ we used

$$
d \tau=d t^{\prime}\left(1-\frac{r^{\prime 2} \omega^{2}}{c^{2}}\right)
$$


Now, Eqs. (7) and (8) coincide each other only if $\omega=\frac{d \phi^{\prime}}{d t^{\prime}}$. But this cannot happen because we consider light propagating in the radial direction, which implies $d \phi^{\prime}=d z^{\prime}=0$. The correct way to proceed is the following. One recalls that $t^{\prime}$ represents the time coordinate in the rotating frame, but, from Eq. (3) it is also $t^{\prime}=t$, where $t$ represents both of the coordinate time and the proper time of the Minkowskian laboratory frame. In a gravitational field, the rate $d \tau$ of the proper time is related to the rate $d t^{\prime}$ of the coordinate time by [21]

$$
d \tau^{2}=g_{00} d t^{\prime 2} .
$$

From the first of Eqs. (3), i.e. $t=t^{\prime}$, one gets immediately from Eq. (9)

$$
d \tau^{2}=g_{00} d t^{2}
$$

Thus, as in a Minkowskian space-time the proper time is equal to the coordinate time, Eq. 10 gives the difference of proper time between the laboratory frame and the rotating frame. as

From Eq. 44 it is $g_{00}=\left(1-\frac{r^{\prime 2} \omega^{2}}{c^{2}}\right)$, and one can, in turn, rewrite Eq. 9

$$
c^{2} d \tau^{2}=\left(1-\frac{r^{\prime 2} \omega^{2}}{c^{2}}\right) c^{2} d t^{\prime 2} .
$$

Then, using again Eq. (3), one gets

$$
c^{2} d t^{\prime 2}=c^{2} d t^{2}=d r^{2}=d r^{\prime 2}
$$

where the equality

$$
c^{2} d t^{2}=d r^{2}
$$

depends on the issue that light propagates in the radial direction in the laboratory frame (the source is indeed a rest in that frame). Thus, one has $d \phi=d z=0$ in Eq. (2) and, by inserting the condition of null geodesics $d s=0$ in the same equation, one immediately obtains Eq. (13).

Hence, Eq. (11) becomes

$$
c^{2} d \tau^{2}=\left(1-\frac{r^{\prime 2} \omega^{2}}{c^{2}}\right) d r^{\prime 2} .
$$

For the sake of correctness, we stress that we considered only radial propagation of light in the rotating system in our previous works [4 - 6]. Actually, this is not a mistake. In fact, despite propagation of light in the rotating frame is not radial, the "gravitational field" has pure radial direction. This implies that the momentum of photons in the rotation direction, which is perpendicular to the radial direction, is conserved. For the same reason, also the momentum of photons in the z-direction is conserved. As a consequence, the two blueshift effects works only in the radial direction in the rotating frame. In fact, despite propagation of light in the rotating frame is not radial, the formula which governs the effect of clock synchronization, i.e. Eq. (14), depends only on the radial coordinate in the rotating frame. 
The root square of Eq. 14 is

$$
c d \tau=\sqrt{1-\frac{r^{\prime 2} \omega^{2}}{c^{2}}} d r^{\prime}
$$

which is equal to Eq. (10) in [4]. Thus, now one can follow step by step the analysis in $[4-6]$.

Eq. (15) is well approximated by [4 - 6]

$$
c d \tau \simeq\left(1-\frac{1}{2} \frac{r^{\prime 2} \omega^{2}}{c^{2}}+\ldots .\right) d r^{\prime}
$$

which gives the second contribution of order $\frac{v^{2}}{c^{2}}$ to the variation of proper time $[4-6]$

$$
c \Delta \tau_{2}=\int_{0}^{r_{1}^{\prime}}\left(1-\frac{1}{2} \frac{\left(r_{1}^{\prime}\right)^{2} \omega^{2}}{c^{2}}\right) d r^{\prime}-r_{1}^{\prime}=-\frac{1}{6} \frac{\left(r_{1}^{\prime}\right)^{3} \omega^{2}}{c^{2}}=-\frac{1}{6} r_{1}^{\prime} \frac{v^{2}}{c^{2}} .
$$

One recalls that $r_{1}^{\prime} \simeq c \tau$ is the radial distance between the source and the detector. Then, one gets the second contribution of order $\frac{v^{2}}{c^{2}}$ to the blueshift as $[4-6]$

$$
z_{2} \equiv \frac{\Delta \tau_{2}}{\tau_{1}}=-k_{2}{\frac{v}{c^{2}}}^{2}=-\frac{1}{6} \frac{v^{2}}{c^{2}} .
$$

Then, one obtains $k_{2}=\frac{1}{6}$ and, using Eqs. (6) and (18), the total blueshift is [4 $-6]$

$$
\begin{aligned}
z & \equiv z_{1}+z_{2}=\frac{\Delta \tau_{10}-\Delta \tau_{11}+\Delta \tau_{2}}{\tau_{1}}=-\left(k_{1}+k_{2}\right) \frac{v^{2}}{c^{2}} \\
& =-\left(\frac{1}{2}+\frac{1}{6}\right) \frac{v^{2}}{c^{2}}=-k \frac{v^{2}}{c^{2}}=-\frac{2}{3} \frac{v^{2}}{c^{2}}=-0 . \overline{6} \frac{v^{2}}{c^{2}},
\end{aligned}
$$

which is completely consistent with the experimental result $k=0.68 \pm 0.03$ in 2. In Eq. 19) $\nabla \tau_{10}$ is the delay of the emitted radiation, $\Delta \tau_{11}$ is the delay of the received radiation and $\Delta \tau_{2}$ is the delay due to clock synchronization.

We can check our computation as it follows. Inserting the condition of null geodesics $d s=0$ in Eq. (4) and considering pure radial motion of photons (as we stressed above, considering only radial propagation of light in the rotating system is not a mistake because of the conservation of the momentum of photons in both the direction of rotation and the z-direction) one gets [4 - 6]

$$
c d t^{\prime}=\frac{d r^{\prime}}{\sqrt{1-\frac{r^{\prime 2} \omega^{2}}{c^{2}}}}
$$

where the positive sign in the square root has been taken, because the radiation is propagating in the positive $r$ direction [4 - 6]. Eq. (20) represents the variation of the coordinate time with respect to the radial coordinate in the rotating frame. But we again recall that, from Eq. (3), one gets also $t^{\prime}=t$, being $t$ both of the 
coordinate time and the proper time $\tau$ of the laboratory frame. Eq. 20 is well approximated by

$$
c d \tau \simeq\left(1+\frac{1}{2} \frac{r^{\prime 2} \omega^{2}}{c^{2}}+\ldots\right) d r^{\prime}
$$

which permits to obtain

$$
c \Delta \tau_{3}=\int_{0}^{r_{1}^{\prime}}\left(1+\frac{1}{2} \frac{\left(r_{1}^{\prime}\right)^{2} \omega^{2}}{c^{2}}\right) d r^{\prime}-r_{1}^{\prime}=+\frac{1}{6} \frac{\left(r_{1}^{\prime}\right)^{3} \omega^{2}}{c^{2}}=+\frac{1}{6} r_{1}^{\prime} \frac{v^{2}}{c^{2}} .
$$

Thus, Eq. 22 shows that, if the rotating observer measures an additional (negative) proper time $\Delta \tau_{2}$ given by Eq. (17), the observer in the laboratory frame measures an additional proper time $\Delta \tau_{3}$ given by Eq. 22 which is

$$
\Delta \tau_{3}=-\Delta \tau_{2}
$$

as one expects.

\subsection{Correspondence with the use of the GTR in Global Positioning Systems}

For the sake of completeness, in this Subsection we review a discussion in 5] on the correspondence between the analysis of the Mössbauer rotor experiment and the use of the GTR in Global Positioning Systems (GPS).

The additional factor $-\frac{1}{6}$ in eq. 18 arises from clock synchronization [5]. This means that its theoretical absence in $[2,3,7-18]$ is due to the incorrect comparison of clock rates between a clock at the origin and one at the detector [5]. This generated wrong claims of invalidity of relativity theory and/or some attempts to explain the experimental results through "exotic" effects [2, 8, 14, 15. 16] which, instead, must be rejected. One of these "exotic" effects will be partially discussed in next Section of this paper.

We used 20] for introducing a discussion of the Langevin metric. This also concerns the use of the GTR in GPS and permits one to realize the following [5]. The additional term $-\frac{1}{6}$ in Eq. 18 is similar to the correction that one has to consider in GPS when one accountes for the difference between the time measured in a frame co-rotating with the Earth geoid and the time measured in a non-rotating Earth centered frame, which is locally inertial [5] (and also the difference between the proper time of an observer at the surface of the Earth and at infinity). In fact, by simply considering the redshift due to the Earth gravitational field, but neglecting the effect of the Earth's rotation, GPS cannot work [5]. Following [5, 20], if one wants to address the problem of clock synchronization within the GPS, one starts from an approximate solution of Einstein's field equations in isotropic coordinates in a locally inertial, nonrotating, freely falling coordinate system with origin at the Earth's center of mass [5, 20]

$$
d s^{2}=\left(1+\frac{2 V}{c^{2}}\right)(c d t)^{2}-\left(1-\frac{2 V}{c^{2}}\right)\left(d r^{2}+r^{2} d \theta^{2}+r^{2} \sin ^{2} \theta d \phi^{2}\right)
$$


where $V$ is the Newtonian gravitational potential of the Earth and $r, \theta, \phi$ are spherical polar coordinates. $V$ is approximately [5, 20]

$$
V \simeq \frac{-G M_{E}}{r}\left[1-J_{2}\left(\frac{a_{1}}{r}\right)^{2} P_{2} \cos \theta\right]
$$

where $M_{E}$ is the Earth's mass, $J_{2}$ the Earth's quadrupole moment coefficient, $a_{1}$ the Earth's equatorial radius and $P_{2}$ the Legendre polynomial of degree 2 [5, 20]. One retains only terms of first order in the small quantity $\frac{V}{c^{2}}$ in Eq. 24. In fact, higher multipole moment contributions to Eq. 25) have negligible effect for relativity in GPS [5, 20]. The equivalent transformations of Eqs. (3) for spherical polar coordinates read [5, 20]

$$
t=t^{\prime}, r=r^{\prime}, \theta=\theta^{\prime}, \phi^{\prime}+\omega_{E} t^{\prime}
$$

where $\omega_{E}$ is the Earth's uniform angular rate. If one applies the transformations (26) to the line element (24) and retains only terms of order $1 / c^{2}$, the line element for the so called Earth-centered, Earth-fixed, reference frame (ECEF frame) is obtained as [5, 20]

$$
\begin{aligned}
d s^{2}= & {\left[1+\frac{2 V}{c^{2}}-\left(\frac{\omega_{E} r^{\prime} \sin \theta^{\prime}}{c}\right)^{2}\right]\left(c d t^{\prime}\right)^{2}+2 \omega_{E} r^{\prime 2} \sin ^{2} \theta^{\prime} d \phi^{\prime} d t^{\prime} } \\
& -\left(1-\frac{2 V}{c^{2}}\right)\left(d r^{\prime 2}+r^{\prime 2} d \theta^{\prime 2}+r^{\prime 2} \sin ^{2} \theta^{\prime} d \phi^{\prime 2}\right) .
\end{aligned}
$$

Standard clocks at rest at infinity define the rate of coordinate time in Eq. 24 [5, 20. Instead, one prefers considering the rate of coordinate time by standard clocks at rest on the Earth's surface [5, 20]. Thus, one introduces a new coordinate time $t^{\prime \prime}$ through a constant rate change [5, 20]:

$$
t^{\prime \prime}=\left(1+\frac{\Phi_{0}}{c^{2}}\right) t^{\prime}=\left(1+\frac{\Phi_{0}}{c^{2}}\right) t
$$

where [5, 20]

$$
\Phi_{0} \equiv-\frac{G M_{E}}{a_{1}}-\frac{G M_{E} J_{2}}{2 a_{1}}-\frac{1}{2}\left(\omega_{E} a_{1}\right)^{2} .
$$

The correction (28) is order seven parts in $10^{10}[5,20$. If one applies this time scale change in the ECEF line element 27 and retains only terms of order $\frac{1}{c^{2}}$, one obtains [5, 20,

$$
\begin{aligned}
d s^{2} & =\left[1+\frac{2\left(\Phi-\Phi_{0}\right)}{c^{2}}\right]\left(c d t^{\prime \prime}\right)^{2}+2 \omega_{E} r^{\prime 2} \sin ^{2} \theta^{\prime} d \phi^{\prime} d t^{\prime \prime} \\
& -\left(1-\frac{2 V}{c^{2}}\right)\left(d r^{\prime 2}+r^{\prime 2} d \theta^{\prime 2}+r^{\prime 2} \sin ^{2} \theta^{\prime} d \phi^{\prime 2}\right)
\end{aligned}
$$

where [5, 20]

$$
\Phi \equiv \frac{V}{c^{2}}-\frac{1}{2}\left(\frac{\omega_{E} r^{\prime} \sin \theta^{\prime}}{c}\right)^{2}
$$


represents the effective gravitational potential in the rotating frame. If one applies the time scale change 28 in the non-rotating line element $(24)$ and drops the primes on $t^{\prime \prime}$ in order to just use the symbol $t$, one gets [5, 20]

$$
d s^{2}=\left[1+\frac{2\left(V-\Phi_{0}\right)}{c^{2}}\right](c d t)^{2}-\left(1-\frac{2 V}{c^{2}}\right)\left(d r^{2}+r^{2} d \theta^{2}+r^{2} \sin ^{2} \theta d \phi^{2}\right) .
$$

The coordinate time $t$ in eq. (32) works in a very large coordinate patch which covers both the Earth and the GPS satellite constellation [5, 20]. Hence, this coordinate time can be used as a basis for synchronization in the Earth's neighborhood [5, 20]. The difference $\left(V-\Phi_{0}\right)$ in the first term of Eq. (32) arises from the following issue [5, 20. In the underlying Earth-centered locally inertial frame where one uses the line element 32 , the unit of time is determined by moving clocks in a spatially-dependent gravitational field [5, 20]. One notices that in Eq. (32) both the effects of apparent slowing of moving clocks and frequency shifts due to gravitation are present [5, 20]. As a consequence, the proper time elapsing on the orbiting GPS clocks cannot be simply used to transfer time from one transmission event to another [5, 20]. Instead, path-dependent effects must be taken into due account [5, 20] in perfect analogy with the discussion of clock synchronization in Subsection 2.2.

We inserted the discussion in this Subsection in order to emphasize that the obtained additional term $-\frac{1}{6}$ in Eq. 18 is not an obscure mathematical or physical detail, but a fundamental ingredient that must be taken into due account $[4-6]$.

\section{Erroneous criticism to our approach}

Our result [4 - 6] on the Mössbauer rotor experiment as new proof of the GTR, that we have reanalysed in previous Section by correcting an important mistake, has been criticized in [17, 18. We stress that such a criticism on our approach does not concern the mistake that we corrected above. Instead, it concerns the issue that, in the opinion of the authors of [17, 18, the Mössbauer rotor experiment cannot detect the second effect of clock synchronization. The key point should be that the extra energy shift due to the clock synchronization is of order $10^{-12} \ldots 10^{-13}$ and cannot be detected by the detectors of $\gamma$-quanta which are completely insensitive to such a very low order of energy shifts [17. In addition, the authors of [17] claim to have shown that the extra energy shift can be explained in the framework of an alternative gravitational theory proposed by themselves. They also insinuate that such a new theory should replace the GTR as the correct theory of gravity [17, 18]. Actually, we have recently show in [22] that the theory proposed in [17, 18] is unscientific because, being a non metric theory, it macroscopically violates EEP, which has today a strong, indisputable, empiric evidence [22, 23]. In addition, in [24] we have also shown that, contrary to the claims in [25], the theory proposed in [17, 18] can reproduce neither the LIGO's "GW150914 signal" nor the other LIGO's detections of gravitational waves. 
Returning to the Mössbauer rotor experiment, in [6] we have shown that in [17. the authors had a misunderstanding of our theoretical analysis in [5. In fact, in [4 -6] and in Subsection 2.2 of the present paper, it has been shown that electromagnetic radiation launched by the central source of the apparatus is shifted of a quantity $0 . \overline{6} \frac{v^{2}}{c^{2}}$ when arriving to the detector of $\gamma$-quanta. Hence, the extra energy shift due to the clock synchronization is of the same order of magnitude of the first effect due to the "gravitational blueshift", as it is obvious if one confronts Eq. (6) with Eq. (18). In fact, in [6] we have shown that, with some clarification, our results in [4 - 6], and, in turn, in Subsection 2.2 of the present paper, hold also when we consider the various steps of the concrete detection. In that case, the resonant absorber detects the energy shift and the separated detector of $\gamma$-quanta merely measures the resulting intensity, see [6] for details. In fact, as we clarified in [6], in [4 -6] and in Subsection 2.2 of the present paper, we assumed as being negligible the difference between the radial coordinate of the resonant absorber and the radial coordinate of the detector of $\gamma$-quanta. In [6] we also clarified the meaning of Eq. (19). It represents the total energy shift that is detected by the resonant absorber as it is measured by an observed located in the detector of $\gamma$-quanta, i.e. located where we have the final output of the measuring [6]. This is different from the total energy shift that is detected by the resonant absorber as it is measured by an observed located in the resonant absorber, which, instead, is given by Eq. (6) 6]. The two quantities should be indeed equal only if the detector of $\gamma$-quanta should be rotating together with the resonant absorber [6]. Instead, the detector of $\gamma$-quanta is fixed [6]. The actual detector (i.e., the receiver of electromagnetic radiation) is the resonant absorber, whose resonant line is shifted with respect to the resonant line of the source in the rotating frame. This induces the variation of intensity of resonant $\gamma$-quanta, passing across this absorber [2]. This intensity is measured by the detector of $\gamma$-quanta, resting outside the rotor system [2]. The latter detector is rather a technical instrument. It allows experimentalist to judge about the shift of the lines of the source and the absorber via the measurement of resonant absorption [2]. But the key point is that the shift of the lines of the source and the absorber that is observed by an observer located in the rotating resonant absorber is different from the shift of the lines of the source and the absorber that is observed by an observer located in the fixed detector of $\gamma$-quanta [6]. That difference is given by the additional factor $-\frac{1}{6}$ in Eq. (18), which comes from clock synchronization. In [6] we also clarified that we are still measuring the total energy shift by using the resonant absorber instead of by using the detector of $\gamma$-quanta as it has been claimed in [17. But the key point is that such a total energy shift measured by an observer located in the fixed detector of $\gamma$-quanta is different from the one measured by an observer located in the rotating resonant absorber [6]. Despite we clarified the above basic issues in [6], the authors of [17] wrote a subsequent paper [18] by claiming that our analysis in [6] was wrong and that the summation of the two components of relative energy shift of our Eq. 190 is definitely inadmissible. Notice that, by making such a claim, the authors of [18 are implicitly stating 
that:

- An apparatus realized to measure a time dilation can measure a particular time dilation but it cannot measure a second time dilation which has the same order of magnitude of the first time dilation. We indeed stress again that the effect of Eq. (6) has the same order of magnitude of the effect of Eq. 18], contrary to the claims in [17].

- The result of a classical (i.e. non-quantum) experiment depends on the way in which the experiment is realized.

Of course, both of the two statements above are completely unscientific. In their attempt to justify such unscientific statements, the authors of [18] claim that our above assertion that "the total energy shift measured by an observer located in the fixed detector of $\gamma$-quanta is different from the one measured by an observer located in the rotating resonant absorber", the we originally stated in [6], is inconsistent from the relativistic viewpoint, and leads to a causal contradiction. They claim indeed that the result of a measurement in Mössbauer rotor experiments is the number of pulses $N$ (i.e., the number of detected resonant $\gamma$-quanta passing through a resonant absorber) during a fixed time interval $T$ at the given tangential velocity $v$ for the absorber. Thus, if one considers an observer co-rotating with the absorber (called the frame $K^{\prime}$ in [18) and a laboratory observer (called $K$ in [18]) attached to the detector placed outside, a strong causal requirement is the equality

$$
N=N^{\prime},
$$

being $N^{\prime}$ the number of pulses in the frame $K^{\prime}$. This means that the indication of a counter of the pulses connected with the output of the detector represents an absolute fact [18. In other words, it must be the same for any observer (including the introduced observers in the frames $K$ and $K^{\prime}$ ) [18. Stating this, the authors of [18] claim that the average number of counts which they define as [18]

$$
\bar{N}=\int_{0}^{T} I(t) d t,
$$

which is obtained via the averaging of the indications of the counter after multiple successive measurements in the frame $K$, would be equal to the average number of counts

$$
\bar{N}^{\prime}=\int_{0}^{T^{\prime}} I^{\prime}\left(t^{\prime}\right) d t^{\prime},
$$

as seen in the frame $K^{\prime}$. In the notations of 18$] I(t)$ and $I^{\prime}\left(t^{\prime}\right)$ are the intensities of the resonant radiation passing through the resonant absorber in the frames $K$ and $K^{\prime}$ respectively, while, in the opinion of the authors of [18, the remaining designations should be obvious. After this, the authors of [18] claim that the 
intensities $I(t)$ and $I^{\prime}\left(t^{\prime}\right)$ can differ from each other due only to the difference of $d t$ and $d t^{\prime}$, which, in their opinion, should be the time dilation effect between the frames $K$ and $K^{\prime}$. Then, the authors of [18] end their analysis by claiming that for the Mössbauer rotor experiment, the latter has a typical difference of the order $10^{-12} \ldots 10^{-13}$ for either rotating or resting observers. Thus, in the opinion of the authors of [18, the admissible range of relative difference between $I(t)$ and $I^{\prime}\left(t^{\prime}\right)$ should entail the same order of magnitude and should be totally negligible [18. Actually, there are a lot of confusion, mistakes and nonsense in the analysis of [18] (that we just reviewed above) which concerns the supposed causal contradiction. Now, we clarify the situation by showing that there is no causal contradiction instead. There are indeed various points to be clarified:

1. By using the notations $d t$ and $d t^{\prime}$ it seems that the authors of [18] used coordinate times rather than proper times in computing the number of pulses in the two different frames. Instead, one must use the proper time. Thus, one must correctly rewrite Eqs. (34) and (35) as

$$
\bar{N}=\int_{0}^{T_{R}} I(\tau) d \tau,
$$

and

$$
\bar{N}^{\prime}=\int_{0}^{T_{L}} I^{\prime}(\tau) d \tau,
$$

respectively, where $T_{R}$ is the proper time which is measured by the rotating observer and $T_{L}$ is the proper time which is measured by the observer at rest in the laboratory. By using Eqs. (6) and (18) one easily gets

$$
T_{R} \simeq \tau_{1}\left(1-\frac{1}{2} \frac{v^{2}}{c^{2}}\right)
$$

and

$$
T_{L} \simeq \tau_{1}\left(1-\frac{1}{2} \frac{v^{2}}{c^{2}}-\frac{1}{6} \frac{v^{2}}{c^{2}}\right)=\tau_{1}\left(1-\frac{2}{3} \frac{v^{2}}{c^{2}}\right),
$$

where we recall that it is $\tau_{1} \simeq \frac{r_{1}^{\prime}}{c}$, being $r_{1}^{\prime} \simeq c \tau_{1}$ the radial distance between the source and the detector, see Subsection 2.1 of this paper.

2. The time dilation effect between the frames $K$ and $K^{\prime}$ is NOT given by the difference of $d t$ and $d t^{\prime}$. In fact, it cannot be an infinitesimal time because, as we stressed in Section 2 of this paper, in a relativistic process of clock synchronization time variations cannot be merely computed by transferring time from one transmission event to another. Instead, pathdependent effects must be taken into due account, see also [5, 20]. Thus, by using Eq. (18), the correct value of the time dilation effect between the frames $K$ and $K^{\prime}$ is given by

$$
T_{L}-T_{R} \simeq-\frac{\tau_{1}}{6} \frac{v^{2}}{c^{2}} .
$$


3. The time dilation effect between the frames $K$ and $K^{\prime}$ is NOT of the order $10^{-12} \ldots 10^{-13}$ (without units!) for either rotating or resting observers as it has been claimed by the authors of [18]. In fact, it is the relative ratio

$$
\frac{T_{L}-T_{R}}{\tau_{1}} \simeq-\frac{1}{6} \frac{v^{2}}{c^{2}},
$$

which is of the order $10^{-12} \ldots 10^{-13}$. Instead the time dilation effect between the frames $K$ and $K^{\prime}$ is of the order

$$
\left|T_{L}-T_{R}\right| \simeq\left|\left(10^{-12} \ldots 10^{-13}\right)\right| \times \tau_{1} .
$$

Now, let us discuss the physical implications of points 1. - 3. The fundamental issue is that, contrary to the claims of the authors of [18, the time dilation effect between the frames $K$ and $K^{\prime}$ is NOT negligible. In fact, the authors of [18] claim that their Mössbauer rotor apparatus detects a total time dilation effect

$$
\triangle \tau \simeq-\frac{2 \tau_{1}}{3} \frac{v^{2}}{c^{2}},
$$

which can be explained through their proper gravitational theory. But this total time dilation effect is of the same order of the time dilation effect between the frames $K$ and $K^{\prime}$, as it is shown by Eq. (40). Thus, if the quantity of Eq. (40) is negligible, also the quantity of Eq. 42 must be negligible, and this merely implies that the Mössbauer rotor apparatus should not work! On the other hand, the issue that the time dilation effect between the frames $K$ and $K^{\prime}$ is not negligible does not imply the presence of causal contradiction. Instead, it merely means that the two different observers in the two different frames $K$ and $K^{\prime}$ measure the same number of pulses in different intervals of proper time. It is exactly this issue which generates the additional effect of clock synchronization. One can also reason as it follows. Let us assume that the authors of [18] are correct and that the difference $T_{L}-T_{R}$ is negligible. Then, in the opinion of the authors of [18] one should find

$$
T_{L} \simeq T_{R} \simeq \tau_{1}\left(1-\frac{2}{3} \frac{v^{2}}{c^{2}}\right),
$$

which is the value predicted by the gravitational theory proposed by the authors of [17, 18] without considering the additional effect of clock synchronization. But, in that case, one can use the same erroneous argument of the authors of [18] concerning the supposed causal contradiction. In fact, in absence of rotation, the number of pulses measured by the observer in the laboratory is given merely by

$$
\bar{N}_{0}=I_{0} \tau_{1},
$$

where $I_{0}$ is the intensity of the resonant radiation passing through the resonant absorber in absence of rotation. Instead, if one actives the rotation, the number of pulses measured by the observer in the laboratory is given by Eq. 36 or 
by Eq. (37), which now coincide because we are assuming that the difference $T_{L}-T_{R}$ is negligible. But, following the ill logic of the authors of of [18, if the quantity

$$
T_{L}-\tau \simeq-\frac{2}{3} \frac{v^{2} \tau_{1}}{c^{2}},
$$

is not negligible, then we should have a causal contradiction because we should obtain $\bar{N}_{0} \neq \bar{N}$. Of course, this is pure nonsense, because the observer in the laboratory measures the same number of pulses in different intervals of proper time in the two different cases of absence or presence of rotation.

All the other criticisms in [18, to our general relativistic approach to the Mössbauer rotor experiment depends on the basic mistake of the authors of 18 concerning the supposed causal contradiction. Thus, also such additional criticisms must be rejected.

We also observe the following. The gravitational theory proposed by the authors of [17, 18] predicts a total value of $\frac{2}{3}$ for the coefficient $k$ in equation (1). But, in [17, 18 the effect of of clock synchronization is not taken into due account. Thus, by considering also this additional effect, if one uses Eq. (18), one gets the correct value of

$$
k=\frac{2}{3}+\frac{1}{6}=\frac{5}{6}
$$

for the gravitational theory proposed by the authors of [17, 18. This result is in contrast with the experimental results in [2]. Thus, contrary to the claims in [17, 18, the theory proposed in such works is completely ruled out by the Mössbauer rotor experiment. We note that the authors of [17, 18] recently published a new work with a further, clumsy attempt to show that our results on the Mössbauer rotor experiment are wrong [30]. The present paper shows that they are the results in [30] which are wrong instead.

\section{Conclusion remarks}

Our results [5, 6] on the Mössbauer rotor experiment representing a new, strong and independent, proof of Einstein's GTR received an Honorable Mention at the Gravity Research Foundation 2018 Awards for Essays on Gravitation 44. In this paper, a mistake which was present in our previous computations [4 -6] on this important issue has been corrected by deriving a rigorous computation of the additional effect of clock synchronization. In the final Section of the paper we have also shown that some recent criticisms on our general relativistic approach to the Mössbauer rotor experiment are incorrect. Thus, the new insights of this paper ultimately confirm our important result.

For the sake of completeness, we stress that, on one hand, our result is also confirmed by a recent result in [26] on the Mössbauer rotor experiment. On the other hand, another recent result in [27] has shown that a general relativistic analysis similar to the one proposed in this paper works also for the Sagnac effect. 


\section{References}

[1] R. L. Mössbauer, Zeitschrift für Physik A (in German) 151, 124 (1958).

[2] A. L. Kholmetskii, T. Yarman, O.V. Missevitch and B. I. Rogozev, Phys. Scr. 79, 065007 (2009).

[3] W. Pauli, Theory of Relativity, Pergamon Press, London (1958).

[4] C. Corda, Int. Journ. Mod. Phys. D 27, 1847016 (2018).

[5] C. Corda, Ann. Phys. 355, 360 (2015).

[6] C. Corda, Ann. Phys. 368, 258 (2016).

[7] W. Kündig, Phys. Rev. 129, 2371 (1963).

[8] A. L. Kholmetskii, T. Yarman and O. V. Missevitch, Phys. Scr. 77, 035302 (2008).

[9] H. J. Hay et al, Phys. Rev. Lett. 4, 165 (1960).

[10] H. J. Hay, in Proc. 2nd Conf. Mössbauer Effect, ed A Schoen and D M T Compton (New York: Wiley) p 225 (1962).

[11] T. E. Granshaw and H. J. Hay, in Proc. Int. School of Physics, 'Enrico Fermi' (New York: Academic) p 220 (1963).

[12] D. C. Champeney and P. B. Moon, Proc. Phys. Soc. 77, 350 (1961).

[13] D. C. Champeney, G. R. Isaak and A. M. Khan, Proc. Phys. Soc. 85, 583 (1965).

[14] A. Kholmetskii, T. Yarman, O. Missevitch, Eur. Phys. Jour. Plus, 128, 42 (2013).

[15] V. O. de Haan, Jour. Comp. Meth. Scien. Eng., 13, 51 (2013).

[16] A. L. Kholmetskii, T. Yarman, M. Arik and O.V. Missevitch, AIP Conf. Proc. 1648, 510011 (2015).

[17] T. Yarman, A. L. Kholmetskii, and M. Arik, Eur. Phys. Jour. Plus 130, $191(2015)$.

[18] A. L. Kholmetskii, T. Yarman, O. Yarman, M. Arik, Ann. Phys. 374, 247 (2016).

[19] C. W. Misner, K. S. Thorne, J. A. Wheeler, "Gravitation", Feeman and Company (1973).

[20] N. Ashby, Liv. Rev. Rel. 6, 1 (2003). 
[21] L. Landau and E. Lifsits - Classical Theory of Fields (3rd ed.). London: Pergamon. ISBN 0-08-016019-0. Vol. 2 of the Course of Theoretical Physics (1971).

[22] C. Corda, Eur. Phys. J. Plus 133, 456 (2018).

[23] C. M. Will, Living Rev. Rel. 17, 4, (2014).

[24] C. Corda, Symmetry 10, 558 (2018).

[25] T. Yarman, A. L. Kholmetskii, O. Yarman, C. B. Marchal, M. Arik, Can. Journ. Phys., 95 (10), 963 (2017).

[26] G. Iovane, E. Benedetto, Ann. Phys. 403, 106 (2019).

[27] E. Benedetto, F. Feleppa, I. Licata, H. Moradpour and C. Corda, Eur. Phys. J. C. 79, 187 (2019).

[28] A. Einstein, Ann. Phys. 49, 769 (1916).

[29] H. Weyl, Raum, Zeit, Materie (Verlag von Julius Springer, Berlin, 1923), 5te Auflage, p.322; see also the 7th edition republished by J.Ehlers in Spinger-Verlag in 1988.

[30] A. L. Kholmetskii, T. Yarman, O. Yarman and M. Arik, Int. Journ. Mod. Phys. D (2019), 10.1142/S021827181950127X 